\title{
Neuropsychological assessments before and after awake surgery for incidental low-grade gliomas
}

\author{
Sam Ng, MD, ${ }^{1}$ Guillaume Herbet, PhD,,-3 Anne-Laure Lemaitre, PhD, ${ }^{1,2}$ \\ Jérôme Cochereau, MD, MSc, ${ }^{3,4}$ Sylvie Moritz-Gasser, PhD, ${ }^{1-3}$ and Hugues Duffau, MD, PhD ${ }^{1-3}$ \\ 1Department of Neurosurgery, Gui de Chauliac Hospital, Montpellier University Medical Center, Montpellier; ${ }^{2}$ Department of \\ Speech-Language Pathology, University of Montpellier; ${ }^{3}$ INSERM U1191, Team "Plasticity of Central Nervous System, Human \\ Stem Cells, and Glial Tumors," Institute of Functional Genomics, Montpellier; and "Department of Neurosurgery, Poitiers \\ University Hospital, Poitiers, France
}

OBJECTIVE Early surgery in presumed asymptomatic patients with incidental low-grade glioma (ILGG) has been suggested to improve maximal resection rates and overall survival. However, no study has reported on the impact of such preventive treatment on cognitive functioning. The aim of this study was to investigate neuropsychological outcomes in patients with ILGG who underwent preventive surgery.

METHODS This was a retrospective analysis of a consecutive series of patients with ILGG who underwent awake surgery and who had presurgical and 3-month postsurgical neuropsychological assessments. Data were normalized into zscores and regrouped by cognitive domains. Clinicoradiological data, histomolecular profile, and differences in z-scores ( $\Delta$ z-scores) were analyzed.

RESULTS Forty-seven patients were included (mean age $39.2 \pm 11.3$ years). Twenty-eight patients (59.6\%) underwent supratotal or total resections. All patients were still alive after a mean follow-up of $33.0 \pm 30.8$ months. Forty-one patients $(87.2 \%)$ had stable $(n=34,72.3 \%)$ or improved ( $\Delta z$-score $>1 ; n=7,14.9 \%)$ neurocognitive outcomes after surgery. Six patients $(12.8 \%)$ presented a slight impairment $(\Delta z$-score $<-1)$ in at least one cognitive domain. The mean presurgical and postsurgical z-scores were comparable except in the psychomotor speed and attention domain. A significant correlation between presurgical executive functioning and tumor volume was reported, whereas the extent of resection and histomolecular profile did not impact neuropsychological outcomes.

CONCLUSIONS Early surgical treatment in presumed asymptomatic patients with ILGG was associated with stable or improved neuropsychological outcomes in $87.2 \%$ of patients at 3 months, with only mild cognitive decline observed in 6 patients. In return, supratotal or total resections were achieved in most patients, and all patients were still alive at the end of the follow-up.

https://thejns.org/doi/abs/10.3171/2020.7.JNS201507

KEYWORDS awake surgery; brain mapping; diffuse low-grade glioma; incidental glioma; neuropsychological assessment; quality of life; oncology; surgical technique

$\mathrm{D}$ IFFUSE low-grade gliomas (LGGs-i.e., WHO grade II gliomas) are frequently discovered incidentally due to increasing access to neuroimaging. Incidental LGGs (ILGGs) precede the symptomatic stage in the natural history of glioma, which is later inevitably characterized by a migration of the tumor and an unpredictable risk of malignant transformation..$^{1-3}$ A considerable improvement in long-term survival has been reported after early and maximal resection in LGG, ${ }^{4-6}$ thereby doubling the overall survival in comparison with a con- servative approach. ${ }^{7}$ Nonetheless, early surgical treatment for ILGG remains a controversial issue, because patients are presumed asymptomatic and may be enjoying active social and professional lives, even though they may suffer from low to moderate neuropsychological disturbances. ${ }^{8}$ Thanks to awake intraoperative mapping, several surgical series have suggested better oncological outcomes due to a greater extent of resection (EOR) in patients with ILGG, ${ }^{9}$ without treatment-related neurological deficits, ${ }^{10}$ with a low rate of postoperative seizures, ${ }^{11}$ and with a high rate

ABBREVIATIONS AED = antiepileptic drug; EOR = extent of resection; ILGG = incidental LGG; LGG = low-grade glioma; RL-RI16 = Rappel libre et Rappel indicé à 16 items; ROCF = Rey-Osterrieth complex figure; TCF = Taylor complex figure; TMT = Trail-Making Test; WAIS-IV = Wechsler Adult Intelligence Scale-Fourth Edition . SUBMITTED April 28, 2020. ACCEPTED July 6, 2020.

INCLUDE WHEN CITING Published online December 4, 2020; DOI: 10.3171/2020.7.JNS201507. 
of return to professional activities. ${ }^{12}$ Although expectations must be set very high in terms of functional results in this subpopulation of LGG, no study to date has investigated neuropsychological outcomes after "prophylactic" surgery. Indeed, neuropsychological functioning is an increasingly important outcome measure in neurooncology ${ }^{13,14}$ because patients with LGG may suffer from deficits in various cognitive domains resulting either from the tumor itself or from current treatment modalities. ${ }^{15,16}$ Such disorders may impact health-related quality of life. ${ }^{17}$ In this study we aimed to assess neuropsychological outcomes by comparing pre- and postoperative cognitive assessments in a consecutive series of patients with ILGG who underwent prophylactic awake surgery.

\section{Methods}

\section{Study Design and Participants}

We conducted a single-center retrospective analysis of a consecutive series of patients with ILGG, who underwent surgery between December 2011 and May 2019. All included patients were asymptomatic (i.e., without clinical manifestations as assessed by a standard neurological examination and without a history of seizures) from initial diagnosis to surgery. Exclusion criteria were as follows: previous chemotherapy or radiotherapy, previous neurological disease with neurocognitive consequences (e.g., multiple sclerosis), non-French-speaking foreigners, and patients lacking perioperative neuropsychological assessments.

\section{Interventions}

Patients were assigned to surgery if 2 or more MRI sessions, spaced at least 3 months apart, demonstrated an objective expansion of FLAIR signal abnormalities. All patients underwent surgery with awake mapping, performed by the senior author (H.D.).

Intraoperative cortical and subcortical mapping was performed using direct electrostimulation, through an asleep-awake-asleep protocol. Intraoperative motor, sensory, and cognitive mapping included counting and DO80 picture naming ${ }^{18}$ in concert with contralateral movement (dual tasks). Complementary tasks were also used, depending on the tumor location, the patient's presurgical language and neuropsychological assessment, and the patient's expectations regarding his/her work and hobbies (e.g., word reading, semantic association, visuospatial tasks, mentalizing). The technical procedures involved with this awake mapping have been extensively described in previous reports. ${ }^{19,20}$ Postsurgical MRI was performed within 24 hours following the surgery and then at 3 months. Antiepileptic drugs (AEDs) were systematically administered during at least the first 3 months following surgery. All patients received intensive cognitive rehabilitation at home after surgery for a period of at least 3 months.

\section{Measures}

Neuropsychological Assessment

All participants underwent a neuropsychological examination the day prior to surgery and 3 months after surgery; the examinations were performed by the same neuropsychologist (G.H. or A.-L.L.). Language abilities were assessed by a dedicated speech therapist (S.M.-G.) in patients with left-sided tumors and/or left-handed patients. The neurocognitive assessment battery was selected to cover all cognitive domains: 1) language and semantics were assessed with the DO80 naming task, ${ }^{18}$ the Pyramids and Palm Trees Test, ${ }^{21}$ and phonological and semantic verbal fluencies; 2) psychomotor speed and attention were assessed using the subtest "code" from the Wechsler Adult Intelligence Scale-Fourth Edition (WAIS-IV), ${ }^{22}$ the subtests "color naming" and "reading" from the Stroop task, ${ }^{23}$ and part A of the Trail-Making Test (TMT); ${ }^{24}$ (3) executive functioning and working memory were assessed using part B of the TMT, phonological and semantic verbal fluencies, Stroop interference minus naming tasks, and forward and backward digit span from the WAIS-IV; 4) episodic verbal memory was assessed with the Rappel libre et Rappel indicé à 16 items (RL-RI16), a French adaptation of the Free and Cued Selective Reminding Test ${ }^{25}$ and 5) visuospatial functioning was assessed with the Rey-Osterrieth complex figure (ROCF), the Taylor complex figure (TCF), ${ }^{26}$ the Bells test, ${ }^{27}$ and the line bisection task. ${ }^{28}$ All participants' raw scores were appropriately aligned to published French normative data (adjusted according to educational level, age, and sex) and subsequently converted into z-scores, except for results from the line bisection tasks, which were converted to a simple cutoff score. The difference between postsurgical z-scores and presurgical z-scores (i.e., $\Delta \mathrm{z}$-score) was calculated.

Note that some additional neuropsychological tasks were tailored to the location of the tumor and to the performances of the patients in a specific domain (e.g., reading of regular words, irregular words, pseudowords, and text reading in right-handed patients with left-sided tumors) and may thus not have been conducted for all patients.

\section{Clinical and Radiological Measures}

The following parameters were obtained: sex, age at the time of surgery, handedness, reason for initial radiological examination, the interval between diagnosis (i.e., the date of the first MRI) and surgery, the follow-up time, and the overall survival.

The tumor volumes (volume at diagnosis, presurgical volume, and postsurgical volume) were calculated using dedicated software (Myrian; Intrasense). The mean velocity of diameter expansion was obtained from the first MRI and the presurgical MRI. The mean diameter $\left(D_{\text {mean }}\right)$ was deduced from the volume $(\mathrm{V})$ by using the previously described formula: ${ }^{29,30} \mathrm{D}_{\text {mean }}=(2 \times \mathrm{V})^{1 / 3}$.

The EOR and residual tumor volume (if any) were measured on the MRI sequences performed 3 months after surgery. In accordance with previous studies, ${ }^{31-33}$ the EOR classification was defined as supratotal when there were no residual FLAIR-weighted signal abnormalities and when the surgical cavity was larger than the presurgical FLAIR tumor volume; as total when no residual FLAIR-weighted signal abnormalities were present; as subtotal when the residual volume was $<10 \mathrm{~cm}^{3}$; and as partial when residual volume was $\geq 10 \mathrm{~cm}^{3}$. In addition, the designation gross-total resection combined both total and supratotal resections. 


\section{Primary and Secondary Outcomes}

Primary Outcome

In order to assess neuropsychological changes before and 3 months after surgery, the following outcomes were analyzed: 1) mean z-scores regrouped by cognitive domain (i.e., language, psychomotor speed and attention, executive functioning, episodic verbal memory, and visuospatial functioning); 2) number of patients who showed stability $(\Delta \mathrm{z}$-score $=[-1 ; 1])$, decline $(\Delta \mathrm{z}$-score $<-1)$, or improvement $(\Delta \mathrm{z}$-score $>1)$ by cognitive domain; and 3 ) number of patients who showed stability in all cognitive domains $(\Delta \mathrm{z}$-score $=[-1 ; 1])$, decline in at least one cognitive domain $(\Delta z$-score $<-1)$, or improvement in at least one cognitive domain $(\Delta \mathrm{z}$-score $>1)$.

Patients with poor cognitive function were considered to have a $\mathrm{z}$-score $<-1$ (indicating that cognitive performance was lower than that of $85 \%$ of the reference population), patients with a deficit were considered to have a zscore $<-1.65$ (indicating that cognitive performance was lower than that of $95 \%$ of the reference population), and patients reporting a variation of $>1 \mathrm{SD}(\Delta \mathrm{z}$-score $<-1$ or $>1$ ) were considered to have a significant change in their performance. A similar methodology has been described in previous studies on this topic. $8,16,34,35$

Note that patients were used as their own control by means of paired presurgical and postsurgical evaluations. This main outcome was designed to specifically assess the effect of surgery on neuropsychological changes, taking into account the possibility that later neuropsychological assessment may have been biased by the progression of the disease itself, or by the administration of adjuvant oncological treatments.

\section{Secondary Outcomes}

Comparisons of $\Delta \mathrm{z}$-scores (regrouped by cognitive domain) were performed according to the hemispheric location of the tumor, the lobal location of the tumor, the type of surgical resection, and histomolecular results. Correlation analyses for each cognitive domain were conducted between mean presurgical z-scores/ $\Delta \mathrm{z}$-scores and tumor characteristics (e.g., age, velocity of diameter expansion, tumor volume).

\section{Standard Protocol Approvals and Patient Consent}

All patients gave informed consent for the retrospective collection of their clinical data. Patients were not subjected to procedures and were not required to follow rules of behavior outside routine clinical care. Data and imaging were analyzed after anonymization in accordance with the Personal Data Protection Act and the Code of Conduct for Responsible Use of Human Tissue and Medical Research. This work was conducted in compliance with the standard ethics of our institution for retrospective study.

\section{Statistical Analysis}

Continuous variables were expressed as the mean \pm $\mathrm{SD}$, and categorical variables were expressed as numbers and proportions. Categorical variables were compared using Fisher's exact test, and continuous variables were compared with either the paired Student t-test or the Wil- coxon signed-rank test, according to the normality of the distribution, which was assessed with the Shapiro-Wilk test. The effect of the side of the tumor on $\Delta z$-scores was assessed using the Mann-Whitney test. The different locations of the tumor, histologies, and types of resection were compared using a 1-way ANOVA or the Kruskal-Wallis test. Spearman's rho correlation coefficient was used to assess the relationship between presurgical z-scores or $\Delta \mathrm{z}$ scores and the following: tumor volume, patients' age, and tumor velocity of diameter expansion. For all statistical analyses, $\mathrm{p}<0.05$ was considered statistically significant. Statistical tests were performed with GraphPad Prism 7.0 (GraphPad Software, Inc.).

\section{Results \\ Participants}

Patient demographics and radiological features are provided in Table 1. The reasons for initial imaging, leading to ILGG diagnosis in most patients, were headache or ear, nose, and throat symptoms $(n=28,59.6 \%)$. All reasons for initial imaging are detailed in Table 2. Forty-seven patients were selected, according to the inclusion criteria (31 women and 16 men), with a mean age of $39.2 \pm 11.3$ years (range 21-65 years) at the time of surgery. The mean delay between image results and surgery was $3.0 \pm 3.62$ years (range $0.17-14.2$ years). The mean presurgical tumor volume was $23.2 \pm 23.9 \mathrm{~cm}^{3}$ (range $1.4-110 \mathrm{~cm}^{3}$ ).

\section{Surgical Results}

Type of resection, EOR, and histomolecular features are provided in Table 1 . Twelve patients $(25.5 \%)$ underwent a supratotal resection, and $16(34.0 \%)$ underwent a total resection. Nineteen patients $(40.4 \%)$ had a subtotal resection, with a mean tumor residual of $1.4 \pm 2.6 \mathrm{~cm}^{3}$ (range 0.4-9 $\mathrm{cm}^{3}$ ). A microfocus with endothelial proliferation was diagnosed in 3 patients $(6.4 \%)$.

\section{Early Postsurgical Outcomes}

Twenty-six patients $(55.3 \%)$ showed a transient immediate postsurgical worsening (including 17 mild language disorders, 3 visuospatial deficits, 3 with hemiparesis, and 3 supplementary motor area syndromes). The mean length of hospital stay was 4.1 days (median 4 days, range 2-7 days). All patients underwent intensive motor and cognitive rehabilitation at home. There were no postsurgical general complications (including hematomas or venous thrombotic events) except for 1 wound infection that required surgical scar revision and antibiotics.

\section{Clinical and Oncological Follow-Up}

Three months after surgery, all patients had totally recovered from transient neurological worsening, assessed by a standard clinical examination. Eight patients $(17.0 \%)$ experienced a transient partial seizure in the postsurgical course, and 3 among them (6.4\% of the cohort) experienced repeated seizures beyond 12 months following surgery. All patients were still receiving AEDs at 3 months after surgery. Thirty patients $(63.8 \%)$ had stopped AEDs at 12 months after surgery. Among 41 patients who were 
TABLE 1. Clinical, radiological, and histopathological features in 47 patients with ILGG

\begin{tabular}{|c|c|}
\hline Feature & $\begin{array}{c}\text { Total Population, } \\
n=47\end{array}$ \\
\hline \multicolumn{2}{|l|}{ Demographics } \\
\hline Mean age, yrs (SD) & $39.2(11.3)$ \\
\hline Sex, female, no. (\%) & $31(66.0)$ \\
\hline Right-handed, no. (\%) & $34(72.3)$ \\
\hline Ambidextrous, no. (\%) & $2(4.3)$ \\
\hline Mean education, yrs (SD) & $14.3(3.1)$ \\
\hline Time from MRI finding to surgery, yrs (SD) & $3.0(3.62)$ \\
\hline \multicolumn{2}{|l|}{ Tumor location, no. (\%) } \\
\hline Left & $27(57.4)$ \\
\hline Right & $20(42.6)$ \\
\hline Frontal & $18(38.3)$ \\
\hline Temporal & $8(17.0)$ \\
\hline Parietal & $4(8.5)$ \\
\hline Insular & $17(36.2)$ \\
\hline \multicolumn{2}{|l|}{ Tumor radiological characteristics } \\
\hline Mean vol at diagnosis, $\mathrm{cm}^{3}(\mathrm{SD})$ & $16.0(20.4)$ \\
\hline Mean preop vol, $\mathrm{cm}^{3}(\mathrm{SD})$ & $23.2(23.9)$ \\
\hline $\begin{array}{l}\text { Mean preop velocity of diameter expansion, } \\
\mathrm{mm} / \mathrm{yr} \text { (SD) }\end{array}$ & $3.5(3.4)$ \\
\hline Mean postop vol, $\mathrm{cm}^{3}(\mathrm{SD})$ & $1.4(2.6)$ \\
\hline Supratotal resection, no. (\%) & $12(25.5)$ \\
\hline Total resection, no. (\%) & $16(34.0)$ \\
\hline Subtotal resection, no. (\%) & $19(40.4)$ \\
\hline Partial resection, no. (\%) & $0(0.0)$ \\
\hline GTR, no. (\%) & $28(59.6)$ \\
\hline \multicolumn{2}{|l|}{ Histopathology, no. (\%) } \\
\hline Microfocus of endothelial proliferation & $3(6.4)$ \\
\hline Diffuse astrocytoma IDH wild-type* & $10(21.3)$ \\
\hline Diffuse astrocytoma IDH mutant* & $13(27.7)$ \\
\hline $\begin{array}{l}\text { Oligodendroglioma IDH mutant/1p19q } \\
\text { codeletion* }\end{array}$ & $23(48.9)$ \\
\hline Oligodendroglioma NOS* & $1(2.1)$ \\
\hline
\end{tabular}

GTR = gross-total resection (calculated as supratotal plus total resections); $\mathrm{IDH}=$ isocitrate dehydrogenase; NOS = not otherwise specified.

* According to the 2016 WHO classification.

employed in normal professional activity before surgery, $38(92.7 \%)$ returned to work on average $5.8 \pm 3.9$ months after surgery (range 1-18 months).

No patient received oncological adjuvant treatment within the first 6 months following surgery. Eleven patients (23.4\%) received chemotherapy on average $30.2 \pm 20.9$ months after surgery (range 8-78 months). Five patients $(10.6 \%)$ received radiotherapy on average $32.0 \pm 19.7$ months after surgery (range 10-48 months). The mean follow-up was $33.0 \pm 30.8$ months (range 3-96 months) after surgery. All patients were still alive at the end of the follow-up.

\section{Presurgical Neuropsychological Assessment}

The results of the baseline neuropsychological testing
TABLE 2. Reasons for initial imaging

\begin{tabular}{lc}
\hline \multicolumn{1}{c}{ Reason for Imaging } & Total Population, $\mathrm{n}=47$ \\
\hline Headache & $19(40.4)$ \\
\hline Head trauma & $3(6.4)$ \\
\hline ENT symptoms & $9(19.1)$ \\
\hline Follow-up for another disease & $2(4.3)$ \\
\hline Research protocol & $1(2.1)$ \\
\hline Screening test for familial history of glioma & $1(2.1)$ \\
\hline Retinal migraine & $3(6.4)$ \\
\hline Anosmia & $2(4.3)$ \\
\hline Facial dysesthesia & $5(10.6)$ \\
\hline Algodystrophy & $1(2.1)$ \\
\hline Meningitis & $1(2.1)$ \\
\hline
\end{tabular}

ENT $=$ ear, nose, throat.

are detailed in Table 3. Episodic memory was the cognitive domain that was most impaired (mean z-score: $-0.48 \pm$ 0.85 ; percentage of patients with z-score $<-1: 30.3 \%$ ). Phonological and categorical fluency task scores were slightly decreased (mean z-scores: $-0.32 \pm 1.20$ and $-0.18 \pm 1.26$, respectively), affecting both language (mean z-score: -0.17 \pm 0.80 ; percentage of patients with z-score $<-1: 10.6 \%$ ) and executive functioning (mean combined z-score: -0.11 \pm 0.67 ; percentage of patients with z-score $<-1: 10.6 \%$ ) domains. Overall, 16 patients $(34.0 \%)$ presented with poor functioning (z-score $<-1$ ) in at least one cognitive domain. Among these, 5 patients $(10.6 \%$ ) presented a deficit (z-score $<-1.65)$ in at least one cognitive domain.

\section{Postsurgical Neuropsychological Assessment at 3 Months and Comparisons With Baseline}

The results of the 3-month postsurgical neuropsychological tests are detailed in Table 3. Additional results including the proportion of patients with z-scores $<-1$ and $>1$ are provided in Supplemental Table 1.

No statistically significant difference was found between presurgical and postsurgical z-scores except in psychomotor speed and attention abilities (mean z-scores: $0.12 \pm 0.72$ vs $-0.22 \pm 1.05$, respectively; $\mathrm{W}=-313 ; \mathrm{p}=$ 0.035 ; Fig. 1). The proportion of patients with cognitive disturbances (z-score $<-1$ ) was comparable before and after surgery within all cognitive domains. No patient presented with significant deviations from normal, either preoperatively or postoperatively, on the line bisection task.

Overall, $87.2 \%$ of patients showed no decline in their cognitive performances $(\Delta z$-score $\geq-1)$ in any cognitive domain at 3 months postsurgery (Fig. 2A). The distribution of patients' $\Delta \mathrm{z}$-scores by cognitive domain is detailed in Fig. 2B. Among 6 patients $(12.8 \%)$ who showed a decline in their cognitive performance $(\Delta \mathrm{z}$-score $<-1)$ in at least one cognitive domain, 4 had an impairment of their psychomotor speed and attention abilities. All 6 patients resumed their professional activities after surgery.

\section{Subgroup Comparisons for $\Delta \mathbf{z}$-Scores}

No impact of lateralization of the tumor on $\Delta \mathrm{z}$-scores 
TABLE 3. Preoperative and 3 months postsurgical neuropsychological test results

\begin{tabular}{|c|c|c|c|}
\hline \multirow[b]{2}{*}{ Test } & \multicolumn{3}{|c|}{ Mean z-Score (SD) } \\
\hline & $\begin{array}{c}\text { Presurgical, } \\
n=47\end{array}$ & $\begin{array}{l}\text { Postsurgical, } \\
n=47\end{array}$ & p Value* \\
\hline \multicolumn{4}{|l|}{ Language } \\
\hline $\mathrm{DO} 80 \dagger$ & $0.13(1.31)$ & $0.19(0.94)$ & \\
\hline PPTT† & $-0.05(1.40)$ & $0.04(1.30)$ & \\
\hline Phonological fluency & $-0.32(1.20)$ & $-0.47(1.09)$ & \\
\hline Categorical fluency & $-0.18(1.26)$ & $-0.37(1.29)$ & \\
\hline Reading text $†$ & $-0.71(0.69)$ & $-0.78(1.06)$ & \\
\hline Regular words $†$ & $-0.07(0.82)$ & $-0.05(0.65)$ & \\
\hline Irregular words $†$ & $0.56(0.59)$ & $0.63(0.57)$ & \\
\hline Pseudowords $†$ & $-0.23(1.15)$ & $-0.26(1.05)$ & \\
\hline Combined z-scores & $-0.17(0.80)$ & $-0.35(0.98)$ & 0.070 \\
\hline \multicolumn{4}{|l|}{$\begin{array}{l}\text { Psychomotor speed and } \\
\text { attention }\end{array}$} \\
\hline WAIS-IV code & $0.29(0.98)$ & $0.14(1.02)$ & \\
\hline TMT A & $0.55(0.55)$ & $0.43(1.05)$ & \\
\hline Stroop naming & $-0.06(1.02)$ & $-0.30(1.41)$ & \\
\hline Stroop reading & $-0.39(1.41)$ & $-0.94(1.57)$ & \\
\hline Combined z-scores & $0.12(0.72)$ & $-0.22(1.05)$ & 0.035 \\
\hline \multicolumn{4}{|l|}{ Executive functioning } \\
\hline TMT B & $0.17(1.01)$ & $0.19(1.46)$ & \\
\hline TMT B-A & $-0.21(1.13)$ & $0.10(0.74)$ & \\
\hline Stroop interference & $-0.13(0.84)$ & $-0.03(0.96)$ & \\
\hline Stroop I-D & $-0.17(0.89)$ & $-0.00(0.94)$ & \\
\hline Phonological fluency & $-0.32(1.20)$ & $-0.47(1.10)$ & \\
\hline Categorical fluency & $-0.18(1.26)$ & $-0.37(1.29)$ & \\
\hline Forward span & $0.05(1.15)$ & $-0.23(1.56)$ & \\
\hline Backward span & $-0.01(0.83)$ & $-0.03(1.08)$ & \\
\hline Combined z-scores & $-0.11(0.67)$ & $-0.11(0.74)$ & 0.967 \\
\hline \multicolumn{4}{|l|}{ Episodic memory } \\
\hline RL-RI16: 1st free recall & $-0.62(1.06)$ & $-0.56(1.48)$ & \\
\hline RL-RI16: 2nd free recall & $-0.37(0.98)$ & $-0.32(0.94)$ & \\
\hline RL-RI16: 3rd free recall & $-0.59(1.03)$ & $-0.59(0.98)$ & \\
\hline RL-RI16: delayed recall & $-0.35(0.96)$ & $-0.80(1.12)$ & \\
\hline Combined z-scores & $-0.48(0.85)$ & $-0.70(1.19)$ & 0.471 \\
\hline \multicolumn{4}{|l|}{ Visuospatial functioning } \\
\hline ROCF/TCF: copy & $0.70(0.49)$ & $0.65(0.50)$ & \\
\hline ROCF/TCF: immediate recall & $0.43(0.85)$ & $0.22(1.07)$ & \\
\hline ROCF/TCF: delayed recall & $0.39(0.95)$ & $0.10(1.14)$ & \\
\hline Bells test: omissions & $0.33(0.98)$ & $-0.07(0.89)$ & \\
\hline Bells test: differences & $0.06(0.82)$ & $0.14(0.68)$ & \\
\hline Combined z-scores & $0.39(0.53)$ & $0.17(0.54)$ & 0.147 \\
\hline
\end{tabular}

PPTT $=$ Pyramids and Palm Trees Test.

* Student t-tests were used to compare combined z-scores for language and executive functioning domains, because variables were normally distributed. Wilcoxon signed-rank tests were used for other cognitive domains, because variables were non-normally distributed.

† Some language tasks were assessed only for patients with left-sided tumors and/or left-handed patients. was found (Fig. 3A). There was, however, a significant effect of tumor location on executive functioning $(\mathrm{H}(2)=$ $6.21, p=0.0448$; Fig. 3B). There was no significant effect of the type of resection (supratotal vs total vs subtotal) on $\Delta \mathrm{z}$-scores in any cognitive domain (Fig. 3C). Similarly, there was no significant effect of histomolecular profile on $\Delta$ z-scores in any cognitive domain (Fig. 3D).

\section{Correlations}

Spearman's rho correlation coefficient in each cognitive domain was used to assess the relationship between presurgical z-scores and tumor volume, patient age, and tumor velocity of diameter expansion. The same method was used to assess the relationship between $\Delta \mathrm{z}$-scores and tumor volume, patient age, and tumor velocity of diameter expansion. Two-tailed tests were conducted. A significant correlation was found between presurgical z-scores in the executive functioning domain and tumor volume $(\mathrm{r}$ $=-0.356[95 \%$ CI $-0.59,-0.068], \mathrm{p}=0.014)$. The presurgical z-score in the language domain and tumor volume showed a tendency to be statistically correlated $(\mathrm{r}=$ -0.281 [95\% CI $-0.53,0.015], \mathrm{p}=0.055)$. The $\Delta \mathrm{z}$-score in the episodic memory domain and patient age also showed a tendency to be statistically correlated $(\mathrm{r}=-0.38[95 \%$ $\mathrm{CI}-0.68,0.020], \mathrm{p}=0.055)$. Other correlations were nonsignificant.

\section{Discussion}

At 3 months after prophylactic surgery for ILGG, standard clinical examinations revealed that all 47 patients had completely recovered from their transient deficits, and additionally, multimodal neurocognitive assessments indicated that $87.2 \%$ of patients presented no significant decline in their higher cognitive functions. Interestingly, nearly $15 \%$ of patients showed an improvement in their cognitive performance, whereas $12.8 \%$ of patients did not show complete recovery of their neuropsychological abilities in the first 3 months following surgery. Overall, psychomotor speed and attention was found to be the cognitive domain that was most impacted by surgery. The strengths of this study include the following: 1) we provide the largest series of patients with LGG who underwent systematic presurgical and postsurgical neuropsychological assessment after awake surgery at a prespecified follow-up time; 2) the patients presented in this series were used as their own controls by means of paired presurgical and postsurgical evaluations; 3) neuropsychological outcomes have not previously been investigated in a selected population of incidentally discovered LGG; and 4) none of the selected patients experienced potential confounding factors (such as seizures) prior to baseline neuropsychological assessment, nor did they receive adjuvant oncological treatment that may have biased postsurgical neuropsychological results. ${ }^{36}$

\section{Preoperative Neuropsychological Assessment}

Many controversies have arisen in the neuro-oncological community on how surgery, chemotherapy, and radiotherapy may contribute to neurocognitive impairments that can have an impact on social life, professional abili- 


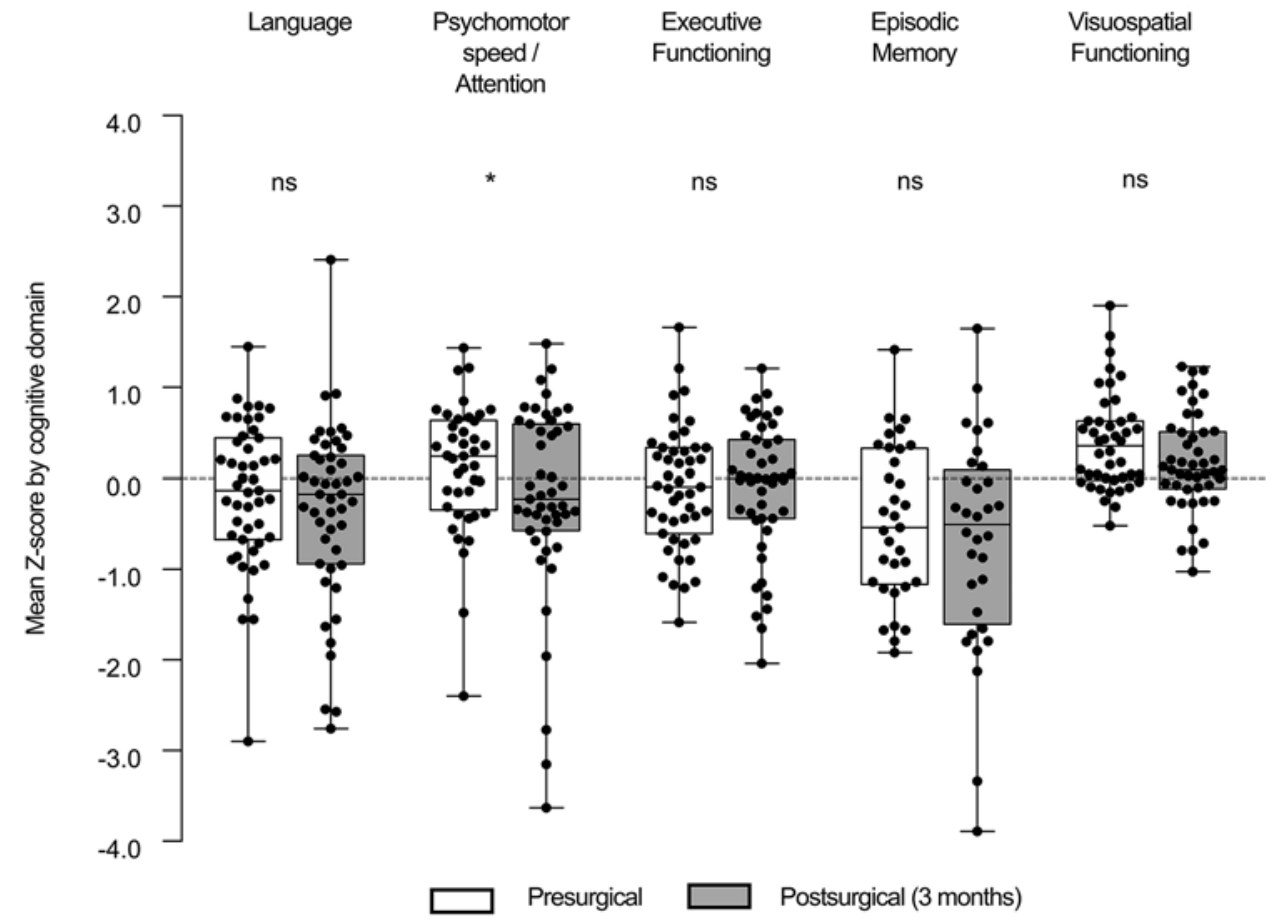

FIG. 1. Box plot showing mean z-scores by cognitive domain: presurgical versus 3-month postsurgical results. Data are presented as medians, quartiles, and extreme values. Student t-tests were used to compare z-scores for language and executive functioning domains, because variables were normally distributed. Wilcoxon signed-rank tests were used for other cognitive domains, because variables were non-normally distributed. $n s=$ nonsignificant. ${ }^{*} p<0.05$.

ties, and consequently health-related quality of life. ${ }^{15}$ As a result of these debates, neuropsychological assessment has emerged as an increasingly important objective measure for evaluation of the impact of current treatment modalities. Pretherapeutic neuropsychological data in ILGG have been rarely reported, but recent studies suggest that most patients who have undergone a cognitive screening, such as the Montreal cognitive assessment, ${ }^{8,16}$ usually suffer from short-term memory loss, reduced attention, and reduced verbal fluency, ${ }^{16}$ with impairments of their psychomotor speed and executive functioning. ${ }^{35}$ Our series supports the finding that more than one-third of patients with ILGG present with poor functioning in at least one cognitive domain, and more than $10 \%$ suffered from an objective cognitive deficit. These results not only confirm that patients with ILGG are not, strictly speaking, asymptomatic, ${ }^{8,16}$ but also suggest that neuropsychological outcomes are related to the natural course of the disease prior to any therapeutic choice. Indeed, presurgical executive functioning deficits were correlated with the volume of the tumor, emphasizing that infiltration of the glioma already impacts cognitive functioning in many so-called presymptomatic patients, ${ }^{37}$ and that symptoms (e.g., seizures) usually occur at an overcompensated stage of brain plasticity. ${ }^{38}$

\section{Postoperative Neuropsychological Assessment}

Recent series reporting functional results after awake surgery for ILGG suggest that complete neurological recovery from transient postoperative deficits, as assessed by a simple clinical examination, is standard at 3 months. ${ }^{9}, 10$
In a recent study investigating cognitive outcomes in a population of 49 adult patients with symptomatic LGG, surgery was even associated with an improvement in memory and executive functions, with no evidence for deterioration in other cognitive domains,$^{34}$ although it is worth mentioning that only 23 patients underwent awake surgery in that series, and that postsurgical neuropsychological assessments were not performed at a prespecified follow-up time. Nevertheless, no study has investigated neuropsychological outcomes in ILGG, although functional outcome measures are categorically required in this context of an incidental discovery. Our results suggest that few patients show an incomplete recovery at 3 months, and that most of these had concerns with psychomotor speed and attention. The variation in performance in psychomotor speed and attention processes must be interpreted with caution, because the mean postsurgical z-score in this domain was $-0.22 \pm 1.05$, which remains close to the score of the general population and may not significantly impact health-related quality of life, especially given that $97.1 \%$ of patients with ILGG were previously reported to have resumed their active professional life after surgery. ${ }^{12}$ Interestingly, correlation analyses indicated that the variation in z-scores induced by surgery is not correlated with the volume of the tumor, suggesting that EOR and neuropsychological outcomes are not related. In addition, subgroup comparisons based on the type of resection (supratotal vs total vs subtotal) did not show significant differences in neuropsychological outcomes, supporting the need to achieve a maximal resection of the tumor for oncological 

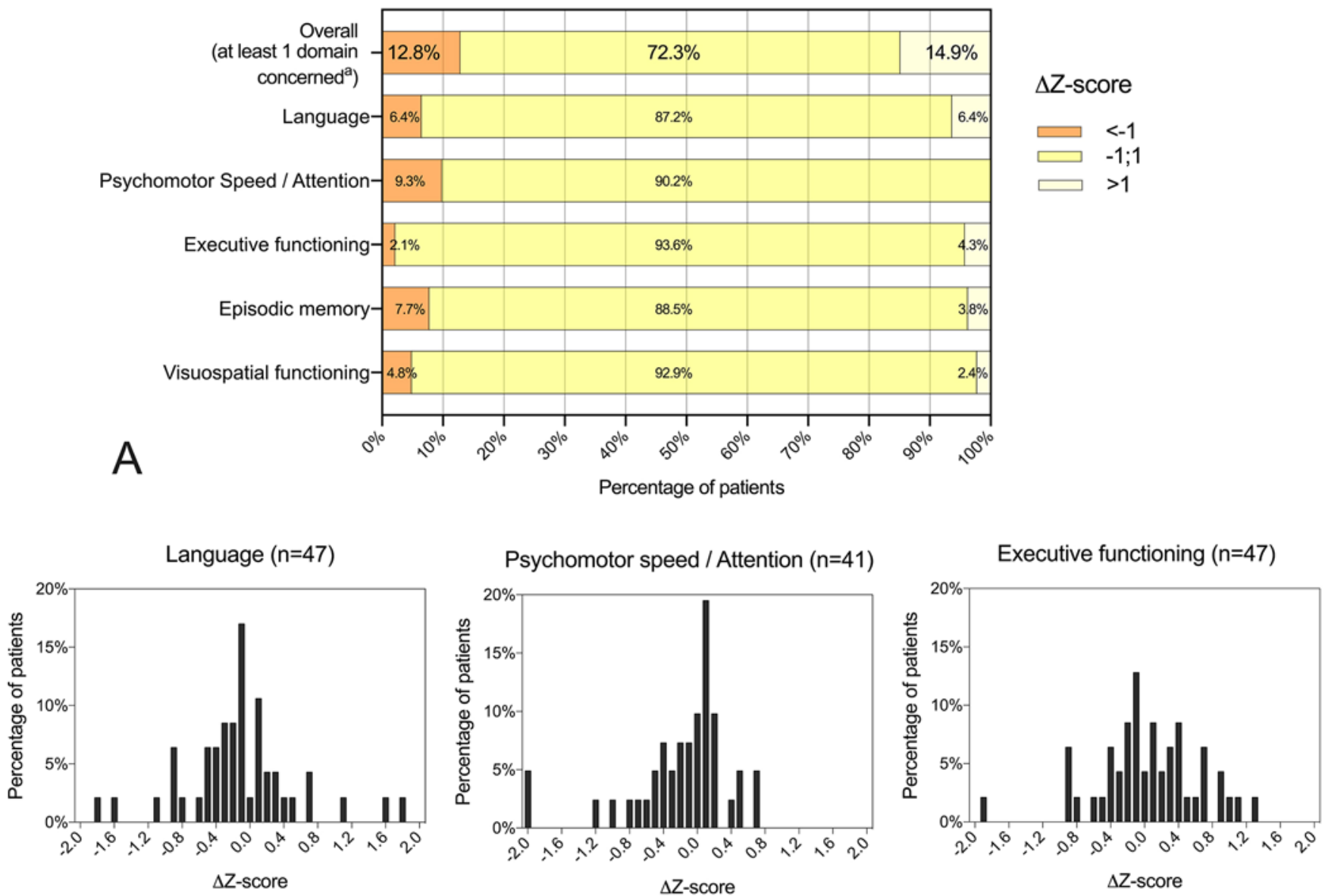

Episodic memory $(n=26)$
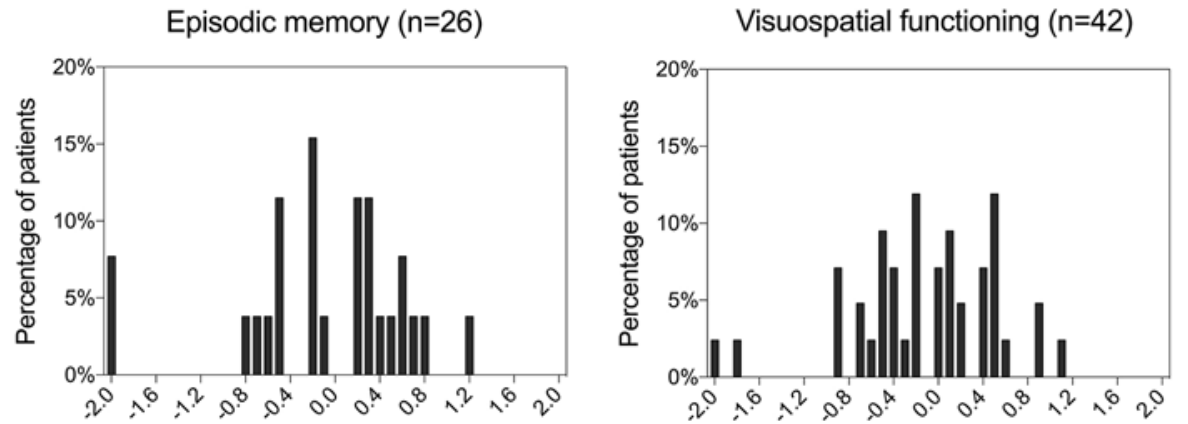

FIG. 2. Graphs showing $\Delta z$-scores at 3-month postsurgical assessment from baseline. A: Percentage of patients with impairment $(\Delta z$-score $<-1)$, stability $(\Delta z$-score $=[-1 ; 1])$, and improvement $(\Delta z$-score $>1)$. B: Distribution of $\Delta z$-scores by cognitive domain. aFor overall score, patients presenting both $\Delta z$-scores $<-1$ and $>1$ in at least one domain were considered as impaired. Reported percentages may not add up to $100 \%$ due to rounding. Figure is available in color online only.

purposes. Interestingly, patient age tended to be correlated with the differences in z-scores after surgery (especially in the episodic memory domain), suggesting that cognitive recovery and neuroplasticity decrease with age.

\section{Rationale for Performing Early Prophylactic Surgery for ILGG}

Although early maximal resection significantly improves overall survival in symptomatic LGG by delaying the risk of malignant transformation, ${ }^{39,40}$ the optimal therapeutic strategy is still open to debate in ILGG, because many neuro-oncologists remain reluctant to propose a prophylactic surgical treatment in so-called asymptomatic patients. The long delay observed in the present study between tumor discovery and surgery (the mean delay was 3 years) is a reflection of this conservative "watch-and-wait" attitude; most patients from this series were diagnosed and followed in other institutions before being referred to our center.

First, ILGG refers to a clinically silent stage belonging to a continuum in the natural history of diffuse glioma (the 

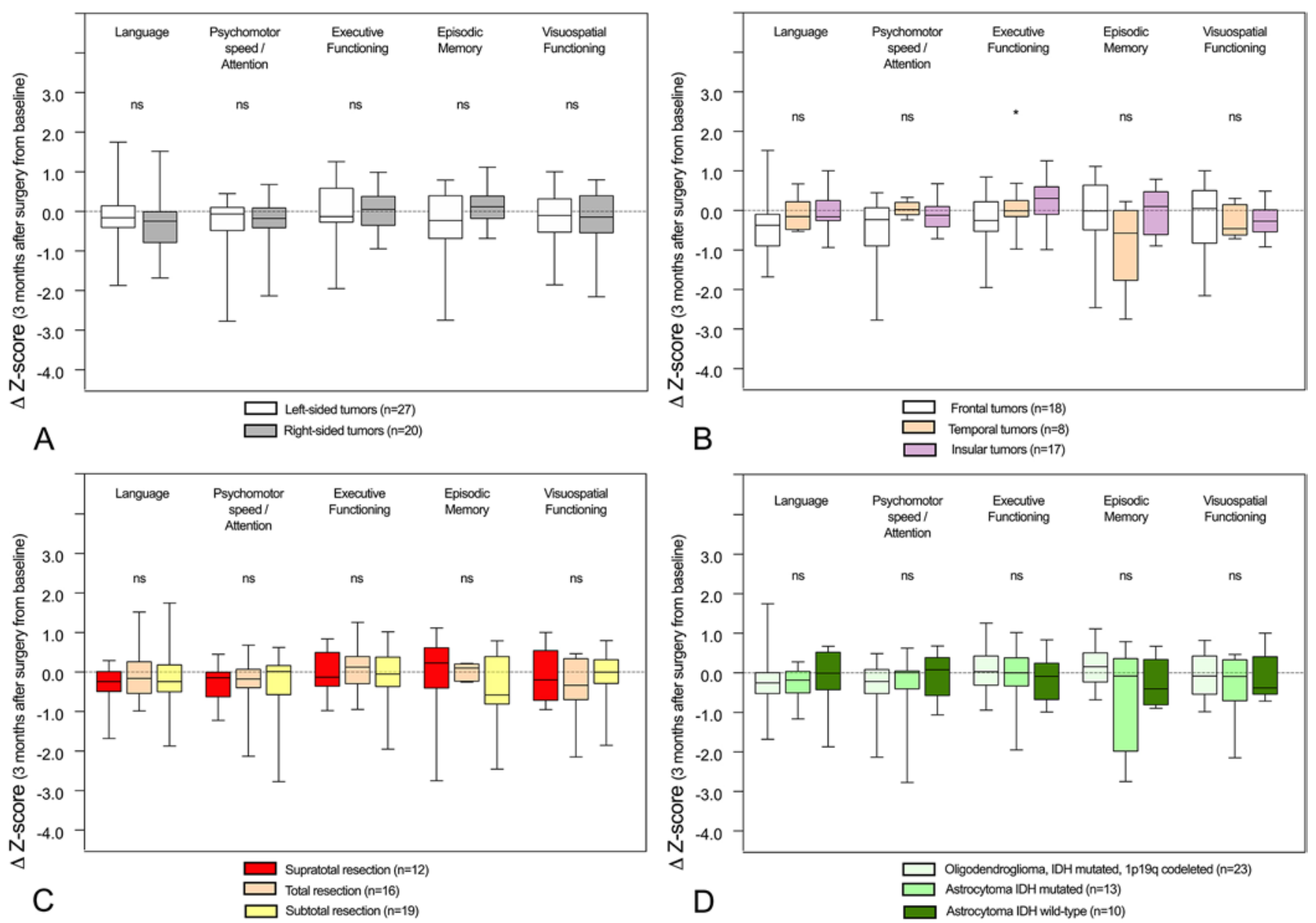

FIG. 3. Box plots showing z-scores and $\Delta z$-scores at 3-month postsurgical assessment from baseline by cognitive domain, based on hemispheric side of the tumor (A), lobe location of the tumor (B), type of resection (C), and histopathological results (D). ${ }^{*} p<$ 0.05. The Mann-Whitney test was conducted to compare hemispheric sides of the tumors. The Kruskal-Wallis test was conducted for other comparisons. Figure is available in color online only.

median delay before reaching the symptomatic stage has been reported to be approximately 48 months $)^{41}$ and shares the same characteristic of permanent migration along the white matter pathways..$^{1,2,29}$ Malignant transformation is unavoidable and unpredictable, with a risk of acute transformation to high-grade glioma at any stage of the disease ${ }^{3}$ (it is worth pointing out that we found 3 patients with a microfocus of endothelial proliferation in this series).

Furthermore, recent studies have reported that EOR was an independent prognostic factor for longer survival in patients with LGG, $4,6,42$ thus advocating for the principle of early resection. In the present series, we report a high rate of supratotal (25.5\%) and total (34.0\%) resections, with limited tumor residuals (mean postoperative volume $1.4 \mathrm{~cm}^{3} ; 0$ partial resections). Indeed, the smaller preoperative tumor volume observed in ILGG allows achievement of higher EOR through preventive surgery and better oncological outcomes, ${ }^{9}$ with the possibility to postpone postoperative adjuvant oncological treatment ${ }^{43}$ (in this series only $11 / 47$ patients received chemotherapy, with a mean delay of 32.0 \pm 20.9 months after surgery; and 5/47 received radiotherapy, with a mean delay of $32.0 \pm 19.7$ months after surgery).
The estimation of the onco-functional balance in incidentally discovered LGG must take into account the oncological benefits reported in the present series (no patients died during a mean follow-up of 33 months) and from other published series. ${ }^{9,16}$ In addition, our results support other studies' findings that awake-guided surgery allows not only the avoidance of permanent neurological deficits but also a high degree of preservation of cognitive performance, with an acceptable rate of mild cognitive decline postsurgery, keeping in mind that such declines may still be compatible with a return to normal daily living and professional activities. ${ }^{12}$ Interestingly, it should also be mentioned that $14.9 \%$ of patients experienced an improvement of their neurocognitive status after surgery. These results will assist medical and surgical neuro-oncologists to better advise patients with newly diagnosed ILGG. In this setting, developing an optimal treatment strategy at the individual level in so-called asymptomatic patients remains challenging, and it is crucial to consider the immediate and delayed consequences of taking a conservative versus a surgical approach. Thus, patients with ILGG must be referred to tertiary centers where they 
can benefit from a personalized therapeutic strategy ${ }^{44}$ tailored to the characteristics of the tumor (volume, location, velocity of expansion, and potential to achieve a total or supratotal resection that would impact the natural course of the disease); to patient demands (their understanding of the concept of a controlled chronic disease and motivation for a prophylactic treatment); and to the socioenvironmental aspects. ${ }^{12}$

Based on the oncological benefits, excellent neurological outcomes, and acceptable cognitive risks observed after so-called prophylactic awake surgery, our results support the implementation of an MRI screening policy in the healthy population, as has already been suggested by some authors. ${ }^{2,45}$ Therefore, a cost-effectiveness analysis and identification of the optimal subpopulation to be screened are now urgently needed.

Our study did have some limitations. First, areas of cognitive performance that were slightly decreased after surgery (mostly psychomotor speed and attention) may be related to an incomplete recovery during the first 3 months following surgery. Later neuropsychological assessments were not systematically performed at a specific endpoint. Second, all patients received AEDs during the first 3 months following surgery, which may have significantly influenced mood and cognition performance. Another limitation is that some neuropsychological tasks were tailored to the location of the tumor. Consequently, patients who were expected to suffer from a specific disturbance (e.g., language abilities in right-handed patients with left-sided tumors) had no missing data for the related neuropsychological tasks, whereas others more frequently had missing data. The introduction of this selection bias may have led to postsurgical results being underrated.

\section{Conclusions}

In this consecutive series of 47 patients with incidentally discovered LGG who underwent early surgery under the awake condition, $87.2 \%$ had stable or improved neurocognitive outcomes at 3 months after surgery. Among them, 7 patients (14.9\%) showed an early improvement of their cognitive abilities in comparison to their presurgical status. Six patients (12.8\%) presented a mild cognitive decline, without consequences for the resumption of their professional activities. In addition, supratotal or total resections were achieved in $59.6 \%$ of patients and all patients were still alive at the end of the follow-up. In light of these favorable oncological outcomes resulting from an early prophylactic surgical management, these novel neurocognitive results may help neuro-oncologists to better estimate the onco-functional balance in patients with newly diagnosed ILGG, in order to devise an optimal and personalized therapeutic strategy.

\section{References}

1. Pallud J, Fontaine D, Duffau H, et al. Natural history of incidental World Health Organization grade II gliomas. Ann Neurol. 2010;68(5):727-733.

2. Mandonnet E, de Witt Hamer P, Pallud J, et al. Silent diffuse low-grade glioma: toward screening and preventive treatment? Cancer. 2014;120(12):1758-1762.
3. Cochereau J, Herbet G, Rigau V, Duffau H. Acute progression of untreated incidental WHO Grade II glioma to glioblastoma in an asymptomatic patient. J Neurosurg. 2016; 124(1):141-145.

4. McGirt MJ, Chaichana KL, Attenello FJ, et al. Extent of surgical resection is independently associated with survival in patients with hemispheric infiltrating low-grade gliomas. Neurosurgery. 2008;63(4):700-708.

5. Schomas DA, Laack NNI, Rao RD, et al. Intracranial lowgrade gliomas in adults: 30 -year experience with long-term follow-up at Mayo Clinic. Neuro Oncol. 2009;11(4):437-445.

6. Capelle L, Fontaine D, Mandonnet E, et al. Spontaneous and therapeutic prognostic factors in adult hemispheric World Health Organization Grade II gliomas: a series of 1097 cases: clinical article. J Neurosurg. 2013;118(6):1157-1168.

7. Jakola AS, Myrmel KS, Kloster R, et al. Comparison of a strategy favoring early surgical resection vs a strategy favoring watchful waiting in low-grade gliomas. JAMA. 2012; 308(18):1881-1888.

8. Cochereau J, Herbet G, Duffau H. Patients with incidental WHO grade II glioma frequently suffer from neuropsychological disturbances. Acta Neurochir (Wien). 2016;158(2):305-312.

9. Ius $\mathrm{T}$, Cesselli $\mathrm{D}$, Isola $\mathrm{M}$, et al. Incidental low-grade gliomas: single-institution management based on clinical, surgical, and molecular data. Neurosurgery. 2020;86(3):391-399.

10. Duffau H. Awake surgery for incidental WHO grade II gliomas involving eloquent areas. Acta Neurochir (Wien). 2012; 154(4):575-584.

11. Lima GL, Duffau H. Is there a risk of seizures in "preventive" awake surgery for incidental diffuse low-grade gliomas? J Neurosurg. 2015;122(6):1397-1405.

12. Ng S, Herbet G, Moritz-Gasser S, Duffau H. Return to work following surgery for incidental diffuse low-grade glioma: a prospective series with 74 patients. Neurosurgery. 2020;87(4):720-729.

13. Taphoorn MJ, Klein M. Cognitive deficits in adult patients with brain tumours. Lancet Neurol. 2004;3(3):159-168.

14. Klein M. Health-related quality of life aspects in patients with low-grade glioma. Adv Tech Stand Neurosurg. 2010;35: 213-235.

15. Klein M. Neurocognitive functioning in adult WHO grade II gliomas: impact of old and new treatment modalities. Neuro Oncol. 2012;14(suppl 4):iv17-iv24.

16. Racine CA, Li J, Molinaro AM, et al. Neurocognitive function in newly diagnosed low-grade glioma patients undergoing surgical resection with awake mapping techniques. Neurosurgery. 2015;77(3):371-379.

17. Jalali R, Dutta D. Factors influencing quality of life in adult patients with primary brain tumors. Neuro Oncol. 2012; 14(suppl 4):iv8-iv16.

18. Metz-Lutz MN, Kremin H, Deloche G, et al. Standardisation d'un test de dénomination orale: contrôle des effets de l'âge, du sexe et du niveau de scolarité chez les sujets adultes normaux. Rev Neuropsychol. 1991;1(1):73-95.

19. Duffau H, Gatignol P, Mandonnet E, et al. New insights into the anatomo-functional connectivity of the semantic system: a study using cortico-subcortical electrostimulations. Brain. 2005;128(Pt 4):797-810.

20. Duffau H. Stimulation mapping of white matter tracts to study brain functional connectivity. Nat Rev Neurol. 2015; 11(5):255-265.

21. Howard D, Patterson K. The Pyramids and Palm Trees Test: A Test of Semantic Access From Words and Pictures. Pearson Assessment; 1992.

22. Doppelt JE, Wallace WL. Standardization of the Wechsler Adult Intelligence Scale for older persons. J Abnorm Psychol. 1955;51(2):312-330.

23. Stroop JR. Studies of interference in serial verbal reactions. $J$ Exp Psychol. 1935;18(6):643-662. 
24. Tombaugh TN. Trail Making Test A and B: normative data stratified by age and education. Arch Clin Neuropsychol. 2004;19(2):203-214.

25. Buschke H. Cued recall in amnesia. J Clin Neuropsychol. 1984;6(4):433-440.

26. Tremblay M-P, Potvin O, Callahan BL, et al. Normative data for the Rey-Osterrieth and the Taylor complex figure tests in Quebec-French people. Arch Clin Neuropsychol. 2015;30(1): 78-87.

27. Gauthier L, Dehaut F, Joanette Y. The Bells test: a quantitative and qualitative test for visual neglect. Int J Clin Neuropsychol. 1989;11(2):49-54.

28. Jewell G, McCourt ME. Pseudoneglect: a review and metaanalysis of performance factors in line bisection tasks. Neuropsychologia. 2000;38(1):93-110.

29. Mandonnet E, Delattre J-Y, Tanguy M-L, et al. Continuous growth of mean tumor diameter in a subset of grade II gliomas. Ann Neurol. 2003;53(4):524-528.

30. Mandonnet E, Pallud J, Clatz O, et al. Computational modeling of the WHO grade II glioma dynamics: principles and applications to management paradigm. Neurosurg Rev. 2008; 31(3):263-269.

31. Berger MS, Deliganis AV, Dobbins J, Keles GE. The effect of extent of resection on recurrence in patients with low grade cerebral hemisphere gliomas. Cancer. 1994;74(6):1784-1791.

32. Yordanova YN, Moritz-Gasser S, Duffau H. Awake surgery for WHO Grade II gliomas within "noneloquent" areas in the left dominant hemisphere: toward a "supratotal" resection. Clinical article. J Neurosurg. 2011;115(2):232-239.

33. Rossi M, Ambrogi F, Gay L, et al. Is supratotal resection achievable in low-grade gliomas? Feasibility, putative factors, safety, and functional outcome. J Neurosurg. 2020;132(6): $1692-1705$

34. Barzilai O, Ben Moshe S, Sitt R, et al. Improvement in cognitive function after surgery for low-grade glioma. J Neurosurg. 2019;130(2):426-434.

35. van Kessel E, Snijders TJ, Baumfalk AE, et al. Neurocognitive changes after awake surgery in glioma patients: a retrospective cohort study. J Neurooncol. 2020;146(1):97-109.

36. McAleer MF, Brown PD. Neurocognitive function following therapy for low-grade gliomas. Semin Radiat Oncol. 2015; 25(3):210-218.

37. Herbet G, Maheu M, Costi E, et al. Mapping neuroplastic potential in brain-damaged patients. Brain. 2016;139(Pt 3): 829-844.

38. Szalisznyo K, Silverstein DN, Duffau H, Smits A. Pathological neural attractor dynamics in slowly growing gliomas supports an optimal time frame for white matter plasticity. PLoS One. 2013;8(7):e69798.

39. Duffau H. Long-term outcomes after supratotal resection of diffuse low-grade gliomas: a consecutive series with 11-year follow-up. Acta Neurochir (Wien). 2016;158(1):51-58.

40. Jakola AS, Skjulsvik AJ, Myrmel KS, et al. Surgical resection versus watchful waiting in low-grade gliomas. Ann Oncol. 2017;28(8):1942-1948.
41. Pallud J, Mandonnet E, Duffau H, et al. Prognostic value of initial magnetic resonance imaging growth rates for World Health Organization grade II gliomas. Ann Neurol. 2006; 60(3):380-383.

42. Cesselli D, Ius T, Isola M, et al. Application of an artificial intelligence algorithm to prognostically stratify grade II gliomas. Cancers (Basel). 2019;12(1):50.

43. Duffau H, Taillandier L. New concepts in the management of diffuse low-grade glioma: proposal of a multistage and individualized therapeutic approach. Neuro Oncol.2015;17(3): 332-342.

44. Duffau H. Paradoxes of evidence-based medicine in lowergrade glioma: to treat the tumor or the patient? Neurology. 2018;91(14):657-662.

45. Kelly PJ. Gliomas: Survival, origin and early detection. Surg Neurol Int. 2010;1(1):96.

\section{Disclosures}

The authors report no conflict of interest concerning the materials or methods used in this study or the findings specified in this paper.

\section{Author Contributions}

Conception and design: Duffau. Acquisition of data: Herbet, Lemaitre, Moritz-Gasser, Duffau. Analysis and interpretation of data: all authors. Drafting the article: $\mathrm{Ng}$. Critically revising the article: Herbet, Lemaitre, Cochereau, Moritz-Gasser, Duffau. Reviewed submitted version of manuscript: Herbet, Lemaitre, Cochereau, Moritz-Gasser, Duffau. Approved the final version of the manuscript on behalf of all authors: $\mathrm{Ng}$. Statistical analysis: $\mathrm{Ng}$, Herbet. Study supervision: Duffau.

\section{Supplemental Information}

Online-Only Content

Supplemental material is available with the online version of the article.

Supplemental Table 1. https://thejns.org/doi/suppl/10.3171/ 2020.7.JNS201507.

\section{Correspondence}

Sam Ng: Gui de Chauliac Hospital, Montpellier University Medical Center, Montpellier, France.s-ng@chu-montpellier.fr. 